\title{
Weiter- und Fortbildung: Ein Kontinuum
}

\section{Hat das Jahr 2017 für das SIWF aussergewöhnliche Herausforderungen gebracht?}

Christoph Hänggeli: Und ob! Die grosse Herausforderung, die wir aufgleisen mussten, war die Neukonstruktion des e-Logbuches. Das aktuelle e-Logbuch erfüllt zwar seine Aufgaben für die Dokumentation der Weiterbildungsanforderungen noch, es lässt sich aber nicht mehr sinnvoll weiterentwickeln, und die Wartung ist extrem aufwendig. Unsere IT-Spezialisten machen dafür die aus heutiger Sicht unzulängliche Software-Architektur verantwortlich, die keine grösseren Veränderungen an den Lernzielkatalogen zulässt. Etwas überspitzt beschrieben, kann beispielsweise bei der Psychiatrie ein Lernziel hinausfallen, wenn man bei den Urologen einen neuen Eingriff einfügt.

\section{Und jetzt?}

Christoph Hänggeli: Alle Analysten sind zum Schluss gekommen, dass die Neuentwicklung eines e-Logbu- ches 2.0 wesentlich rascher und kostengünstiger ist als die Reparatur des bestehenden Systems. Die von uns betrauten Experten versichern, dass die neue, modular aufgebaute Version über eine viel besser strukturierte Programmierung verfügt, die auch künftigen Anforderungen voll genügen wird. Zunächst fallen aber hohe Kosten an, die das SIWF über den Abbau seiner finanziellen Reserven tragen kann. Allerdings müssen wir den Gürtel für einige Jahre enger schnallen und andere Projekte zurückstellen.

Giatgen Spinas: Ich möchte ergänzen, dass die Funktion des e-Logbuchs für die Weiterzubildenden absolut zentral ist und dass es klaglos funktionieren muss. Es wird in Zukunft ermöglichen, Titelgesuche noch rascher zu bearbeiten, und es wird auch wichtige statistische Informationen liefern, unter anderen die Grundlagen für die Finanzierung der Weiterbildungsstellen durch die Kantone.

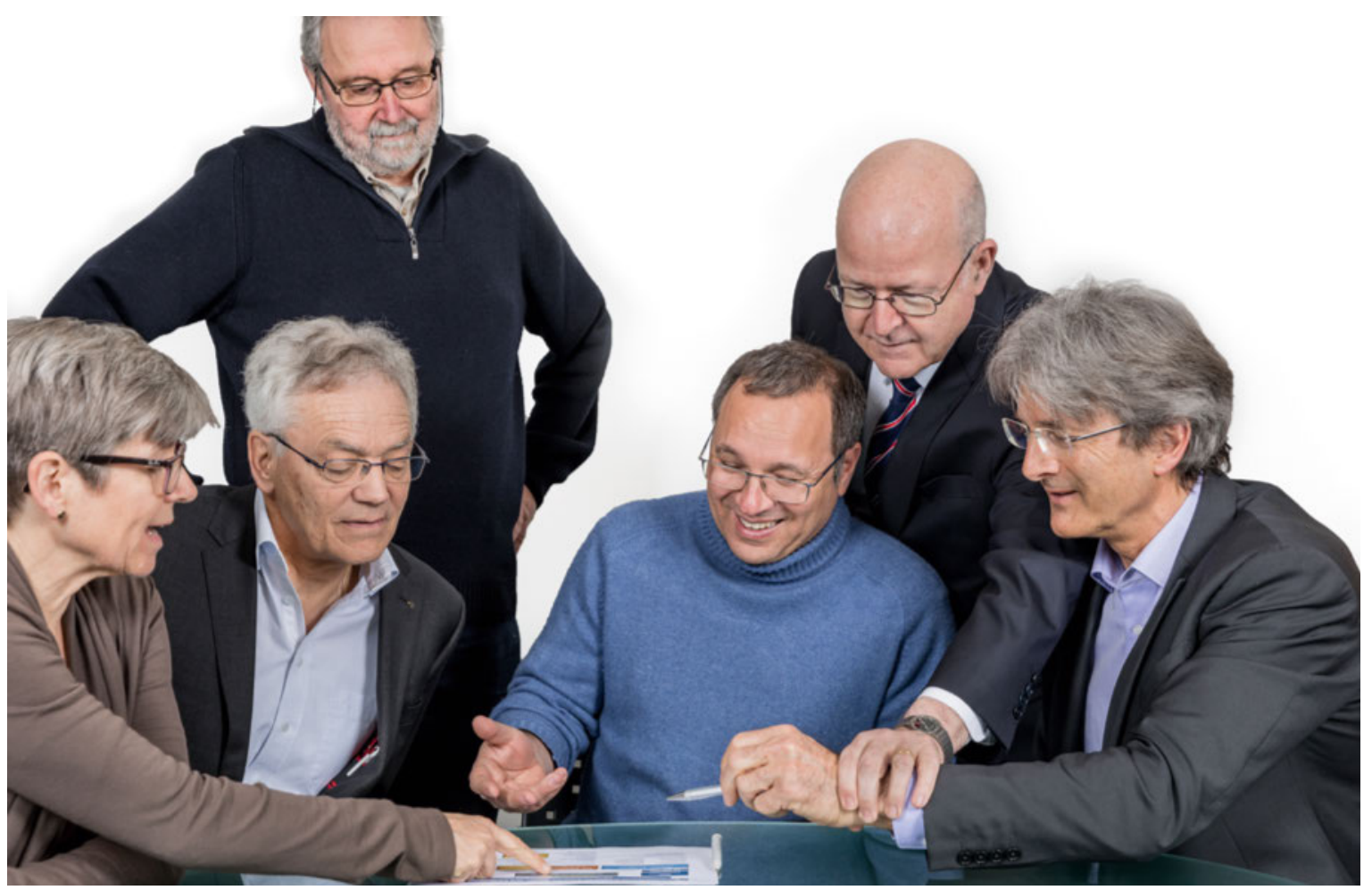

Die Geschäftsleitung des SIWF: Regula Schmid, Giatgen A. Spinas, Jean Pierre Keller, Raphael Stolz, Werner Bauer und Christoph Hänggeli (v.I.n.r.) 
Was gibt es über das vergangene Jahr Erfreuliches zu berichten?

Werner Bauer: Grundsätzlich ist das schweizerische Weiterbildungsmodell erfolgreich, was bei vielen Auslandkontakten zum Ausdruck gebracht wird. Ein Beispiel sind die Visitationen, die wir beim Wechsel in der Leitung der Weiterbildungsstätte durchführen und die Gegenstand grossen Interesses sind. Im Moment läuft die Akkreditierung der Weiterbildung durch das Departement des Inneren, mit welcher unserem Institut alle sieben Jahre das Mandat zur Erteilung der eidgenössischen Facharzttitel erneuert wird. Dabei können Auflagen formuliert werden. Soweit wir informiert sind, sind die Feststellungen der Experten grundsätzlich positiv.

\section{Das Weiterbildungsmodell der Schweiz beruht auf einer Kombination von Dienstleistung und Lernen. Entstehen da keine Konflikte?}

Regula Schmid: Dieses Modell hat eine lange Tradition und erfüllt so lange seine Aufgabe zur Zufriedenheit, als an den Weiterbildungsstätten die Voraussetzungen dafür gegeben sind: Die Assistenzärztinnen und Assistenzärzte müssen vom ärztlichen Kader genügend angeleitet und überwacht werden. Die Erfahrungen am Patientenbett, im Operationssaal oder in der Sprechstunde bilden die entscheidende Basis für den Aufbau der notwendigen Kompetenzen. Ohne motivierte Weiterbildende und genügend Zeit geht es nicht.

Werner Bauer: Sie fragen nach Konflikten: Es gibt sie und sie verschärfen sich. Die Rahmenbedingungen für die Bildung werden schlechter, weil in den Spitälern die Anforderungen an die Effizienz und die administrativen und ökonomischen Belastungen für die Kaderärzte laufend zunehmen. Zudem ist Zeit natürlich Geld, und die Finanzierung der Weiterbildung ist leider bei weitem noch nicht befriedigend sichergestellt. Es braucht deshalb gerade heute ein besonderes Engagement von uns allen im Bewusstsein, dass das Weitergeben der notwendigen Kompetenzen an die nächste Ärztegeneration für das Gesundheitswesen absolut essenziell ist.
Korrespondenz: Schweizerisches Institut für ärztliche Weiter und Fortbildung SIWF FMH

Elfenstrasse 18

CH-3000 Bern 15

Tel. 0313591111

siwf[at]fmh.ch

\section{Könnten neue didaktische Methoden die Weiter- und Fortbildung unterstützen?}

Giatgen Spinas: Bis zu einem gewissen Grad wird das möglich sein. Gegenwärtig sind Simulation und Robotik in voller Entwicklung. Schon heute können mit Unterstützung von Simulatoren sehr gute, auch interdisziplinäre Fortbildungen in den Fächern Anästhesie, Intensiv-

\section{Vollständige Fassung SIWF-Geschäftsbericht}

Die vollständige Fassung des SIWF-Geschäftsberichts inklusive Bericht «SIWF Kompakt» und einem Rückblick zur Geschäftsstelle des SIWF finden Sie auf der Website des SIWF unter: www.siwf.ch $\rightarrow$ Über uns $\rightarrow$ Geschäftsberichte. medizin und auch Notfallmedizin durchgeführt werden. Robotik und Simulation werden auch einen Beitrag an die Bewältigung der zunehmenden Schwierigkeit leisten, eine genügende Anzahl von Fällen für das Erlernen von operativen Eingriffen zur Verfügung zu haben.

\section{Hat das SIWF einen Einfluss darauf, wie viele Fach-} ärzte in den verschiedenen Disziplinen weitergebildet werden?

Jean Pierre Keller: Diese Frage wird lebhaft diskutiert, auch im Hinblick auf das Verhältnis von Hausärzten zu Spezialärzten. Die Fachgesellschaften und das SIWF können die Zahl zukünftiger Fachärzte indirekt über die Anforderungen in den Weiterbildungsprogrammen beschränkt beeinflussen. Die Frage, ob bedarfsgerechte Planung und sinnvolle Steuerung möglich und wünschbar ist, ruft eher skeptische Reaktionen hervor.

\section{Das SIWF hat auch einen Auftrag im Bereich der Fortbildung. Nimmt es diesen mit der Fortbildungs- plattform wahr?}

Raphael Stolz: Ja, aber nicht nur. Jede Fachgesellschaft hat ein Fortbildungsprogramm, das auf der Basis der SIWFFortbildungsordnung erstellt worden ist und das den speziellen Aspekten der Disziplin gerecht werden kann. Die Fortbildungsplattform ist ein problemlos funktionierendes Instrument, um die Fortbildung zu dokumentieren und von den Fachgesellschaften bestätigen zu lassen, worauf das Fortbildungsdiplom ausgedruckt werden kann. Auch wenn der Arztberuf ein selbstverantwortlicher, akademischer Beruf ist, können wir nicht darüber hinwegsehen, dass die Fortbildung eine Pflicht ist und dass von Patienten, Versicherern und Politikern erwartet wird, dass sie glaubhaft erfüllt und nachgewiesen wird.

\section{Sehen Sie neue Entwicklungen? Man spricht ja gelegentlich von einem Kontinuum Aus-Weiter-Fort- bildung.}

Werner Bauer: Dieses Kontinuum wird immer wichtiger, und seine Realisierung stellt eine grosse Herausforderung dar. In Zukunft wird wohl das Tätigkeitsspektrum bei der Pensionierung sehr verschieden von demjenigen beim Absolvieren der Facharztprüfung sein. Der Kompetenzerhalt und das Erlernen neuer Kompetenzen werden eine zusätzliche Strukturierung und Evaluation benötigen. International spricht man deshalb auch kaum mehr von Fortbildung, sondern von "continuing professional development". Die Arbeit wird dem SIWF nicht ausgehen.

Bildnachweis

Tobias Schmid/SIWF 